\title{
Anti-Sway System for Ship-to-Shore Cranes
}

\author{
Edvin Raubar ${ }^{*}$ - Damir Vrančić2 \\ ${ }^{1}$ Luka Koper, d.d., Slovenia \\ 2 Jožef Stefan Institute, Slovenia
}

\begin{abstract}
All ship-to-shore cranes hoisting mechanisms are made of a load attached to the trolley by means of a hoisting rope. In the process of loading and unloading the ship, the trolley moves the load along the boom from ship to shore and vice versa. During movement, the hoisting mechanism structure causes swinging of the load around vertical position. Load swing increases the loading/unloading time and increases the probability of collisions with other objects, like a nearby container, an iron construction for disposal of material (e.g. bunker), etc. Swinging of the load cannot be avoided, but can be radically reduced by using appropriate anti-sway systems.

The paper derives a non-linear and linearized dynamic model of the crane load based on parameters of Panamax ship-to-shore crane in the Port of Koper. The responses of three open-loop anti-sway systems are compared together on the aforementioned dynamic model. Simulation results show that all three systems reduce load swing significantly, but only the systems based on zero-vibration-derivative and zero-vibration-derivative-derivative methods completely satisfy the given requirements.
\end{abstract}

Keywords: ship-to-shore crane, load oscillation, anti-sway system, open-loop system

\section{O INTRODUCTION}

Permanent increase of productivity of ship-to-shore cranes in Port of Koper is very important in order to remain competitive with other North Adriatic Sea ports. Increasing productivity can be achieved by reducing trans-shipment time. One possible way to increase productivity is to use anti-sway systems, which can efficiently reduce load oscillations. A reduction of oscillations increases the speed of transshipment and consecutively the ship-to-shore crane productivity.

The paper derives a dynamic mathematical model of the crane load with accompanying limitations. The three open loop anti-sway systems are compared together on the aforementioned dynamic model with parameters taken from technical specification of Panamax ship-to-shore crane in Port of Koper.

The cranes do not have anti-sway system integrated, so the operator must wait until the load oscillation is completely cancelled before positioning the load to the desired position on the ship or on the truck. The maximum acceptable deviation during positioning the load on the trucks with the ship-toshore cranes in the Port of Koper is $0.1 \mathrm{~m}$, which corresponds to angular deviation of $0.01 \mathrm{rad}\left(0.57^{\circ}\right)$.

Generally, anti-sway systems are divided into two main groups: the open-loop and the closed-loop systems (see Figs. 1 and 2). The closed-loop systems are based on feedback information of the current load angular deviation, trolley position and its velocity (which are measured by additional sensors). The open-loop systems operate by applying feed-forward actions. They foresee error and try to eliminate it before it occurs [1].

In this paper, the open-loop systems will be tested on a model of Panamax ship-to-shore crane in Port of Koper.

The paper consists of three sections. In the first section, a non-linear and linearized mathematical model of the ship-to-shore crane load oscillation is derived. In the second section, three anti-sway systems are presented. In the third section the performance and

\begin{tabular}{|c|c|c|c|c|c|c|}
\hline $\begin{array}{c}\text { Desired force } \\
\text { on the trolley }\end{array}$ & $\begin{array}{c}\text { Crane load } \\
\text { anti-sway system }\end{array}$ & \begin{tabular}{|c|} 
Shaped input force \\
on the trolley
\end{tabular} & $\begin{array}{c}\text { Regulated force } \\
\text { on the trolley }\end{array}$ & $\underset{\text { position }}{\stackrel{\text { Trolley }}{\longrightarrow}}$ & Load & $\underset{\text { response }}{\text { System }}$ \\
\hline
\end{tabular}

Fig. 1. Block diagram of open loop (feed-forward) anti-sway system

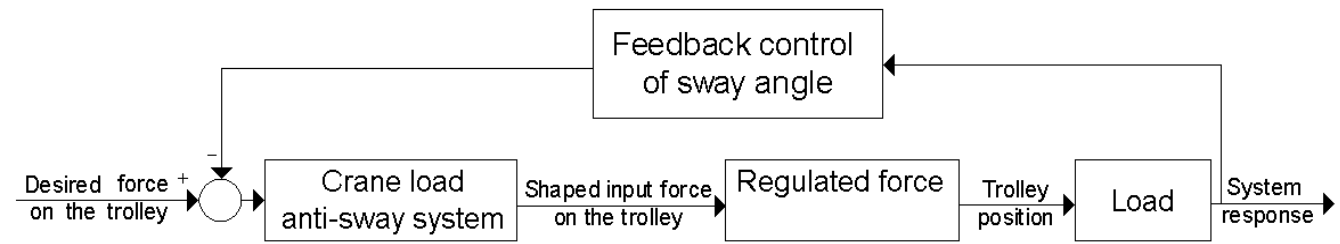

Fig. 2. Block diagram of closed loop anti-sway system 
time responses of all three methods are compared and evaluated. Conclusions are given in section four.

\section{MODELING}

The trolley (see Fig. 3) can travel only when an external force is applied through the hoisting rope. The force accelerates or decelerates the trolley. The friction force between steel wheels and the rail is very small compared to other forces, so it is neglected.

The length of the hoisting rope is time-invariant function, since the operator does not move the trolley and hoist at the same time. The length of the hoisting rope also does not depend upon load mass. The trolley and the load can be considered as point masses, which move in two dimensions only ( $x-y$ coordinate plane).

The applied force to the trolley is managed by the operator. By using the joystick, the operator defines the desired trolley velocity. The information about the desired trolley velocity is sent to frequency inverter, which controls the speed of motors. Motors, by means of gears, wind or unwind the rope on the drum and create force on the trolley. This force is positive when the trolley is accelerating and negative when the trolley is decelerating.

The force, which accelerates or decelerates the trolley, is always the same in magnitude, but changes direction, which depends on the desired velocity.

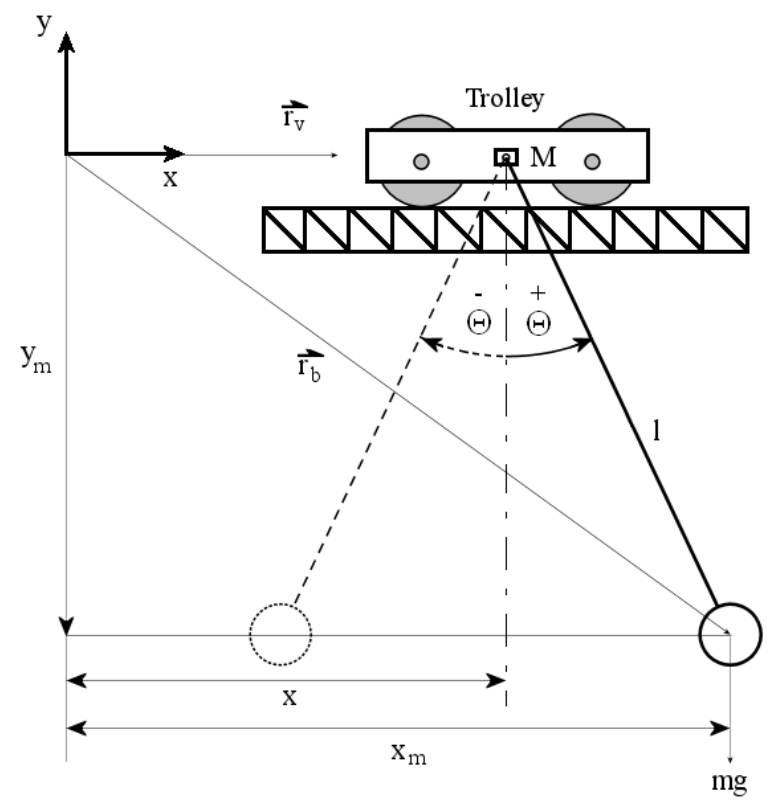

Fig. 3. Model of ship-to-shore crane load and trolley

During trolley acceleration the load moves away from the balance position clockwise. In this case the angular deviation $\Theta$ has a negative sign. During trolley deceleration, the load moves anticlockwise. In this case the angular deviation $\Theta$ has a positive sign (see Fig. 3).

In the sequel the following variables will be used: $x \quad$ trolley horizontal position [m],

$\dot{x} \quad$ trolley velocity $[\mathrm{m} / \mathrm{s}]$,

$\Theta \quad$ load angular deviation [rad],

$\dot{\Theta} \quad$ load angular velocity $[\mathrm{rad} / \mathrm{s}]$,

$l \quad$ length of the hoisting rope [m],

$M \quad$ mass of the trolley $[\mathrm{kg}]$,

$m \quad$ mass of the payload $[\mathrm{kg}]$,

$g \quad$ gravitational acceleration $\left[\mathrm{m} / \mathrm{s}^{2}\right]$.

\subsection{Mathematical Model}

According to Fig. 3, the trolley position vector $\vec{r}_{v}$ and load position vector $\vec{r}_{b}$ are defined as:

$$
\begin{gathered}
\vec{r}_{b}=(x+l \sin \Theta,-l \cos \Theta), \\
\dot{\vec{r}}_{b}=(\dot{x}+l \dot{\Theta} \cos \Theta, l \dot{\Theta} \sin \Theta), \\
\vec{r}_{v}=(x, 0), \\
\dot{\vec{r}}_{v}=(\dot{x}, 0) .
\end{gathered}
$$

The horizontal position $x$ is limited between -17 and $37 \mathrm{~m}$. Initial or parking position is defined as $x=$ 0 and $\Theta=0$.

Initial coordinates of vectors $\vec{r}_{v}$ and $\vec{r}_{b}$ are:

$$
\begin{gathered}
\vec{r}_{b 0}=(0,-l), \\
\vec{r}_{v 0}=(0,0) .
\end{gathered}
$$

The kinetic and potential energy of the load are described by the following expressions [1]:

$$
\begin{gathered}
W_{k}=W_{v}+W_{b}=\frac{1}{2} M \dot{\dot{r}_{v}} \cdot \dot{\overrightarrow{r_{v}}}+\frac{1}{2} m \dot{\overrightarrow{r_{b}}} \cdot \dot{\overrightarrow{r_{b}}}= \\
=\frac{1}{2} M \dot{x}^{2}+\frac{1}{2} m\left(\dot{x}^{2}+l^{2} \dot{\Theta}^{2}+2 l \dot{x} \dot{\Theta} \cos \Theta\right), \\
W_{p}=m g y_{m}=-m g l \cos \Theta .
\end{gathered}
$$

By using Lagrangian function [1], the secondorder non-linear model can be derived as follows:

$$
\ddot{x}=\frac{F_{x}+m g \cos \Theta \sin \Theta+m l \dot{\Theta}^{2} \sin \Theta}{\left(M+m-m \cos ^{2} \Theta\right)},
$$

$\ddot{\Theta}=\frac{-F_{x} \cos \Theta-m l \dot{\Theta}^{2} \cos \Theta \sin \Theta-M g \sin \Theta-m g \sin \Theta}{l\left(M+m \sin ^{2} \Theta\right)}$. 


\subsection{Linearized Model}

Eqs. (9) and (10) describe nonlinear model. The nonlinear model could be linearized by using certain assumptions [1]. The first assumption is that load swing angle is small during trolley movement. Based on that assumption the expansion of sine and cosine functions can be done by using the first term of Taylor series:

$$
\begin{aligned}
& \sin \Theta=\Theta-\frac{\Theta^{3}}{3 !}+\frac{\Theta^{5}}{5 !}-\frac{\Theta^{7}}{7 !}+\ldots \approx \Theta, \\
& \cos \Theta=1-\frac{\Theta^{2}}{2 !}+\frac{\Theta^{4}}{4 !}-\frac{\Theta^{6}}{6 !}+\ldots \approx 1 .
\end{aligned}
$$

Taking Eqs. (11) and (12) into Eqs. (9) and (10), we get two differential equations with two variables:

$$
\begin{gathered}
\ddot{\Theta}=-\left[\left(\frac{M+m}{M l}\right) g \Theta+\frac{F_{x}}{M l}\right], \\
\ddot{x}=\left(\frac{m}{M}\right) g \Theta+\frac{F_{x}}{M} .
\end{gathered}
$$

\subsection{Validation of the Linearized Model}

In order to validate the linearized model, the responses of linearized and non-linear model to the same input signal have been compared by means of simulation. Input signal is generated by step functions, which represent force on the trolley. Simulation parameters are given in Table 1. They correspond to the actual parameters of Panamax ship-to-shore crane in Port of Koper.

Table 1. Parameters used in simulation

\begin{tabular}{lcc}
\hline Mass of the trolley & $M$ & $25 \mathrm{t}$ \\
\hline Mass of the payload & $m$ & $30 \mathrm{t}$ \\
\hline Length of the hoisting rope & $I$ & $10 \mathrm{~m}$ \\
\hline Max trolley velocity & $v V$ & $2 \mathrm{~m} / \mathrm{s}$ \\
\hline Max acceleration of trolley & $a v$ & $0.3 \mathrm{~m} / \mathrm{s}^{2}$ \\
\hline Gravitational acceleration & $g$ & $9.81 \mathrm{~m} / \mathrm{s}^{2}$ \\
\hline Force on the trolley & $F x$ & $20 \mathrm{kN}$ \\
\hline
\end{tabular}

From Fig. 4 it can be seen that the trolley is not travelling uniformly after the force is taken off, since attached load is swinging with its own natural frequency. This causes slight acceleration or deceleration of the trolley (depends on angular deviation $\Theta$ ). It can be seen that there are very small differences between linearized and nonlinear model. The assumptions in Eqs. (11) and (12) are therefore, due to small swing angles, correct.
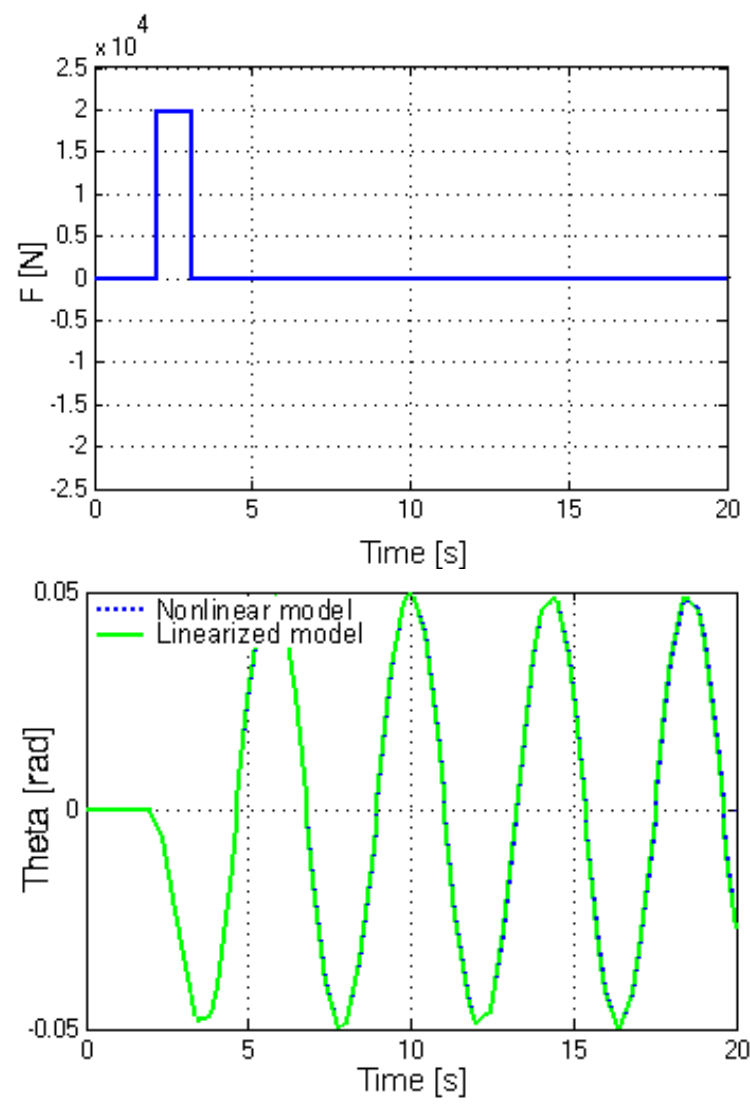

Fig. 4. System response to impulse input excitation signal

\section{ANTI-SWAY SYSTEM FOR SHIP-TO-SHORE CRANES}

\subsection{Main Working Principles}

All the methods, which will be used for the reduction of oscillation, are generating an input signal that cancels its own oscillation. The simplest method is the so-called Zero-Vibration (ZV) Shaper that consists of two impulses. The first impulse, which starts the system oscillating, is located at time zero, and the second impulse is delayed by half period of the oscillation. The oscillation caused by the second impulse is out of phase with the first oscillation, thereby cancelling it (see Fig. 6) [2] .

The input signal can be shaped with any number of impulses. However, the amplitudes and time instants should be derived from the system's natural frequencies, damping ratios and the following set of constraints [2]:

- $\quad$ after the last impulse is applied, the oscillations must be cancelled (zero residual vibration constraints),

- the sum of amplitudes must be equal to one (unity magnitude summation constraints), 
- the derivative of the second-order system response on the Nth impulse must be zero (robustness constraints),

- time instants of impulses must be calculated so as to get minimum system response delay (time optimality constraints).

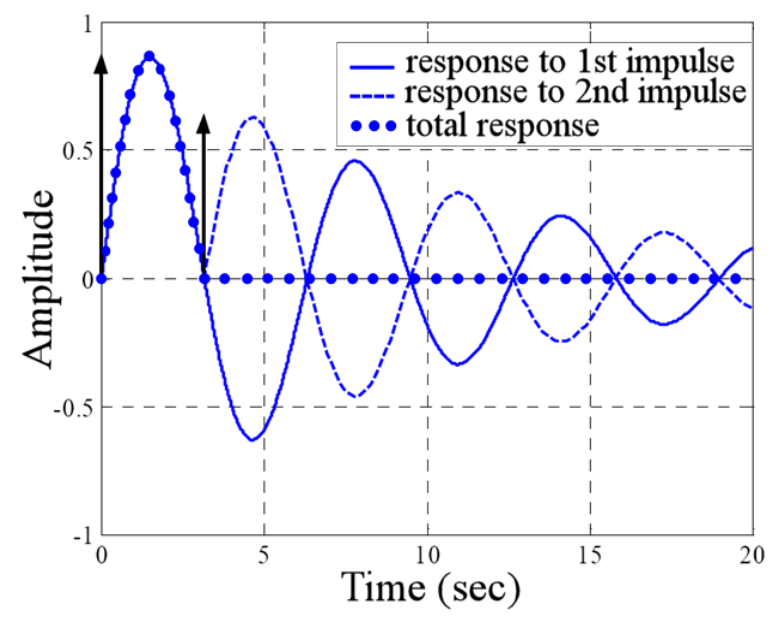

Fig. 5. System response with two impulses; first impulse generates oscillations, while the second impulse cancels them out

\subsection{Mathematical Formulation of the Constrained Equations}

The following equation describes the impulse response of the second-order underdamped system [3]:

$$
y(t)=\left[A \frac{\omega_{0}}{\sqrt{1-\xi^{2}}} e^{-\xi \omega_{0}\left(t-t_{0}\right)}\right] \cdot \sin \left(\omega_{0} \sqrt{1-\xi^{2}}\left(t-t_{0}\right)\right),
$$

where $A$ is the amplitude of the impulse, $t_{0}$ is the impulse time, $\omega_{0}$ is underdamped natural frequency and $\xi$ is damping ratio. The second-order system response on $N$ impulses could be written as [4] and [5]:

$$
\begin{gathered}
y(t)=\sum_{j=1}^{N} B_{j} \sin \left(\omega_{0} \sqrt{1-\xi^{2}}\left(t-t_{j}\right)\right), \\
A_{\text {amp }}=\sqrt{V_{1}^{2}+V_{2}^{2}}, \\
V_{1}=\sum_{j=1}^{N} B_{j} \cos \theta_{j}, \\
V_{2}=\sum_{j=1}^{N} B_{j} \sin \theta_{j}, \\
B_{j}=A_{j} \frac{\omega_{0}}{\sqrt{1-\xi^{2}}} e^{-\xi \omega_{0}\left(t_{N}-t_{j}\right)},
\end{gathered}
$$

$$
\theta_{j}=\omega_{0} \sqrt{1-\xi^{2}} t_{j}
$$

where $A_{\text {amp }}$ is the multi-impulse vibration amplitude of the response and is obtained at the instant of the last impulse, $t_{N}$. $B_{j}$ is the coefficient which determines the amplitude of sine function [2].

To obtain zero vibration after the last impulse, the Eq. (17) must be zero. This happens when Eqs. (18) and (19) are independently zero. With this assumption the first two constrained equations are obtained:

$$
\begin{aligned}
& V_{1}=\sum_{j=1}^{N} A_{j} \frac{\omega_{0}}{\sqrt{1-\xi^{2}}} e^{-\xi \omega_{0}\left(t_{N}-t_{j}\right)} \cos \left(\omega_{0} \sqrt{1-\xi^{2}} t_{j}\right)=0, \\
& V_{2}=\sum_{j=1}^{N} A_{j} \frac{\omega_{0}}{\sqrt{1-\xi^{2}}} e^{-\xi \omega_{0}\left(t_{N}-t_{j}\right)} \sin \left(\omega_{0} \sqrt{1-\xi^{2}} t_{j}\right)=0 .
\end{aligned}
$$

An additional assumption is that the summation of impulse amplitudes must be equal to one and the amplitudes should be positive values:

$$
\sum_{i=1}^{N} A_{i}=1
$$

When the nonlinear system contains higher sinusoidal harmonics (as in our case), it is necessary to use more impulses to efficiently reduce the oscillation, which request more equations to be solved. In this case the robustness constraints are used that increase system accuracy (and thus stability) forcing higher derivatives of functions toward zero [2]:

$$
\begin{aligned}
& \frac{d^{q} V_{1}}{d \omega_{0}^{q}}=\sum_{j=1}^{N}\left(\begin{array}{l}
A_{j}\left(t_{j}\right)^{q} \frac{\omega_{0}}{\sqrt{1-\xi^{2}}} e^{-\zeta \omega_{0}\left(t_{N}-t_{j}\right)} \cdot . \\
\cdot \cos \left(\omega_{0} \sqrt{1-\zeta^{2}} t_{j}\right)
\end{array}\right)=0, \\
& \frac{d^{q} V_{2}}{d \omega_{0}^{q}}=\sum_{j=1}^{N}\left(\begin{array}{l}
A_{j}\left(t_{j}\right)^{q} \frac{\omega_{0}}{\sqrt{1-\xi^{2}}} e^{-\zeta \omega_{0}\left(t_{N}-t_{j}\right)} \cdot \\
\cdot \sin \left(\omega_{0} \sqrt{1-\zeta^{2}} t_{j}\right)
\end{array}\right)=0 .
\end{aligned}
$$

System stability increases by increasing the order (q) of derivatives.

To get minimal system response delay, the first impulse must be applied at time origin:

$$
t_{1}=0 \text {. }
$$

\subsection{Methods for Shaping Input Signal}

In this section three methods for shaping input signal are presented. These methods are frequently used in practice [6] to [9]: 
- Zero-Vibration Shaper (ZV)

- Zero-Vibration-Derivative Shaper (ZVD)

- Zero-Vibration-Derivative-Derivative Shaper (ZVDD).

The main difference between the methods is the number of impulses applied for shaping input signal. ZV method is using two, ZVD three and ZVDD four impulses.

The amplitudes and time instants of impulses can be calculated from Eqs. (17) to (27) [2]. The amplitudes and time instants of $\mathrm{ZV}$ shaper are the following:

$$
\begin{gathered}
A_{1}=\frac{1}{1+K}, \quad t_{1}=0, \\
A_{2}=\frac{K}{1+K}, \quad t_{2}=\frac{\pi}{\omega_{0} \sqrt{1-\zeta^{2}}}, \\
K=e^{-\frac{\zeta \pi}{\sqrt{1-\zeta^{2}}}} .
\end{gathered}
$$

The amplitudes and time instants of ZVD shaper are, correspondingly, the following:

$$
\begin{gathered}
A_{1}=\frac{1}{1+2 K+K^{2}}, \quad t_{1}=0, \\
A_{2}=\frac{1}{1+2 K+K^{2}}, \quad t_{2}=\frac{\pi}{\omega_{0} \sqrt{1-\zeta^{2}}}, \\
A_{3}=\frac{3 K^{2}}{1+3 K+3 K^{2}+K^{3}}, \quad t_{3}=\frac{2 \pi}{\omega_{0} \sqrt{1-\zeta^{2}}}, \\
K=e^{-\frac{\zeta \pi}{\sqrt{1-\zeta^{2}}}} .
\end{gathered}
$$

The amplitudes and time instants of ZVDD shaper are as follows:

$$
\begin{gathered}
A_{1}=\frac{1}{1+3 K+3 K^{2}+K^{3}}, \quad t_{1}=0 \\
A_{2}=\frac{3 K}{1+3 K+3 K^{2}+K^{3}}, \quad t_{2}=\frac{\pi}{\omega_{0} \sqrt{1-\zeta^{2}}}, \\
A_{3}=\frac{3 K^{2}}{1+3 K+3 K^{2}+K^{3}}, \quad t_{3}=\frac{2 \pi}{\omega_{0} \sqrt{1-\zeta^{2}}}, \\
A_{4}=\frac{K^{3}}{1+3 K+3 K^{2}+K^{3}}, \quad t_{4}=\frac{3 \pi}{\omega_{0} \sqrt{1-\zeta^{2}}},
\end{gathered}
$$

$$
K=e^{-\frac{\zeta \pi}{\sqrt{1-\zeta^{2}}}}
$$

Table 2 shows the amplitudes and times of impulses using ZV, ZVD and ZVDD methods based on calculated system natural frequency and damping ratio (see Table 1)

Table 2. Amplitudes and time instants of impulses using ZV, ZVD in ZVDD method

\begin{tabular}{rcc}
\hline Method & Amplitude of impulses & Time instant of impulses [s] \\
\hline \multirow{2}{*}{ ZV } & $A_{1}=0.5$ & $t_{1}=0$ \\
\cline { 2 - 3 } & $A_{2}=0.5$ & $t_{2}=1.78$ \\
\hline \multirow{3}{*}{ ZVD } & $A_{1}=0.25$ & $t_{1}=0$ \\
\cline { 2 - 3 } & $A_{2}=0.5$ & $t_{2}=1.78$ \\
\cline { 2 - 3 } & $A_{3}=0.25$ & $t_{3}=3.56$ \\
\hline \multirow{3}{*}{ ZVDD } & $A_{1}=0.125$ & $t_{1}=0$ \\
\cline { 2 - 3 } & $A_{2}=0.375$ & $t_{2}=1.78$ \\
\cline { 2 - 3 } & $A_{3}=0.375$ & $t_{3}=3.56$ \\
\cline { 2 - 3 } & $A_{4}=0.125$ & $t_{4}=5.34$ \\
\hline
\end{tabular}

\section{SIMULATION OF TROLLEY AND LOAD MOVEMENT USING ANTI-SWAY SYSTEM}

This section simulates performance of different anti-sway systems during trolley acceleration and deceleration using the non-linear mathematical model presented in Section 1. In the simulation, ZV, ZVD and ZVDD shapers are used.

The input signal in the simulation represents force on trolley. First, the positive impulse is applied which accelerates the trolley for the first 5 seconds (see Fig. 6). In the next 5 seconds the trolley is traveling uniformly without acceleration. Then, the force is applied in the opposite direction causing the trolley to uniformly decelerate until it stops.

The shaped input signals (the actual forces), when applying ZV, ZVD and ZVDD shapers, are shown in Fig. 7. Trolley positions and angles are shown in Fig. 8.

It can be seen that anti-sway systems noticeably reduce load angular deviation and the amplitude of oscillations in the steady-state. On the the other hand, the anti-sway systems slightly increase settling time.

Considering the requirement for maximum load angular deviation given in the Introduction, it can be seen that the ZV shaping method does not satisfy the requirements, since load angular deviation in the steady-state is higher than $0.01 \mathrm{rad}$.

In comparison to the ZV shaping method, the ZVD shaping method has better performance. The highest load angular deviation in the steady-state 
is now $0.0045 \mathrm{rad}\left(0.26^{\circ}\right)$. Settling time (when the angular deviation becomes lower than $0.01 \mathrm{rad}$ ) is achieved at 17.75 or $2.75 \mathrm{~s}$ after the input force stops acting to the trolley (Fig. 6).

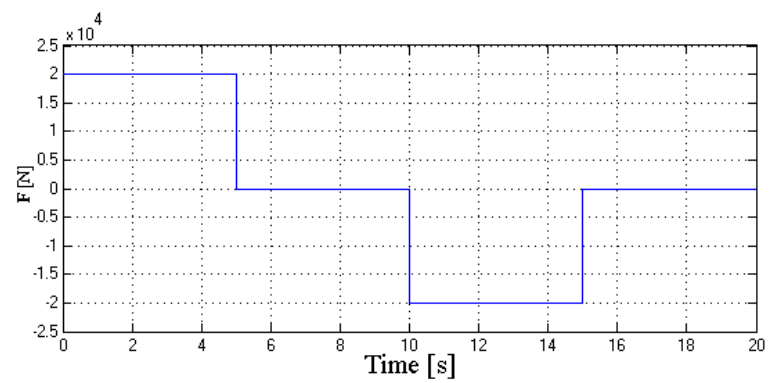

Fig. 6. Force to the trolley
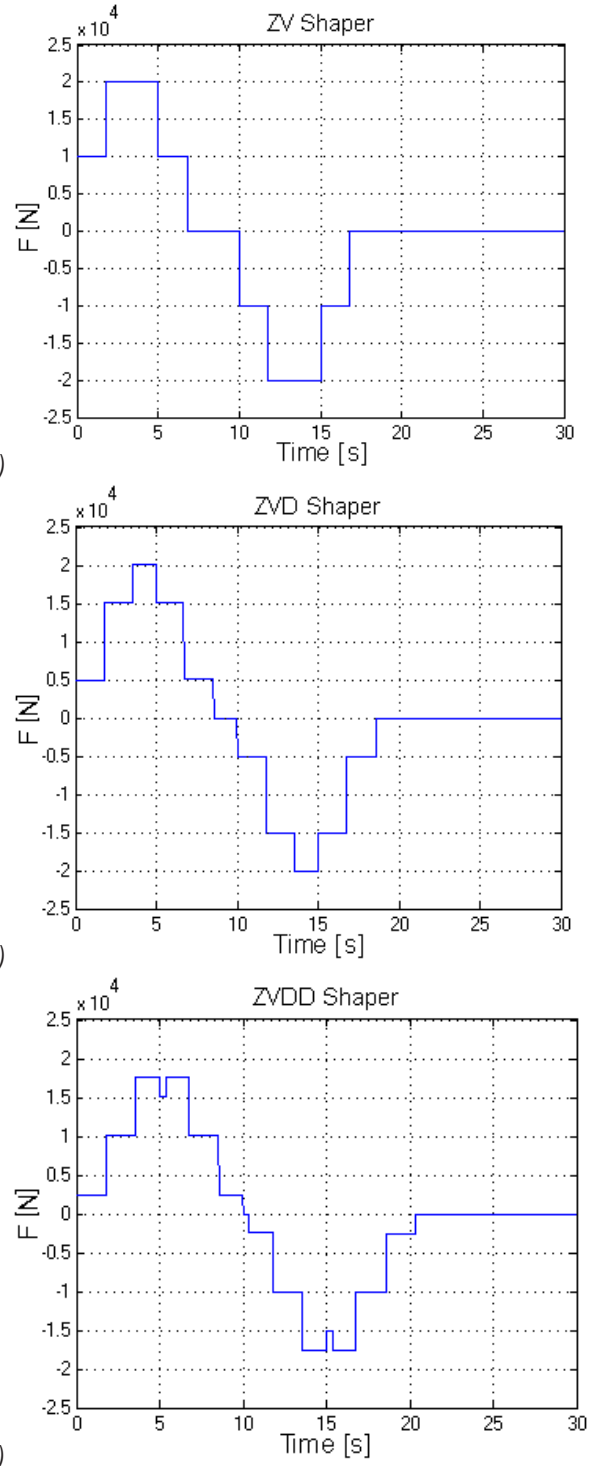

Fig. 7. Shaped input force signal, a) ZV shaper, b) ZVD shaper, c) ZVDD shaper
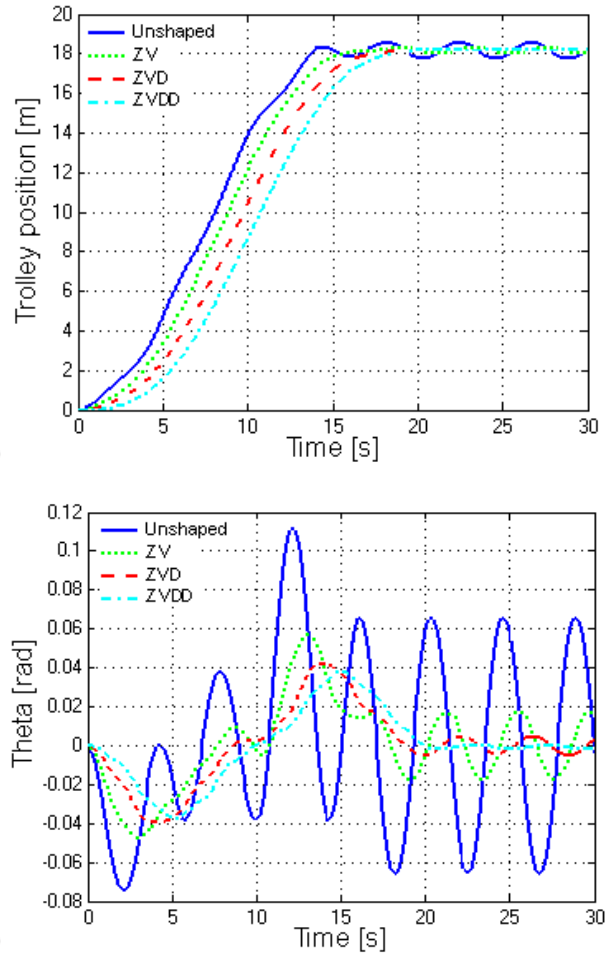

Fig. 8. a) Trolley position and b) load angle with ZV, ZVD and ZVDD Shaper
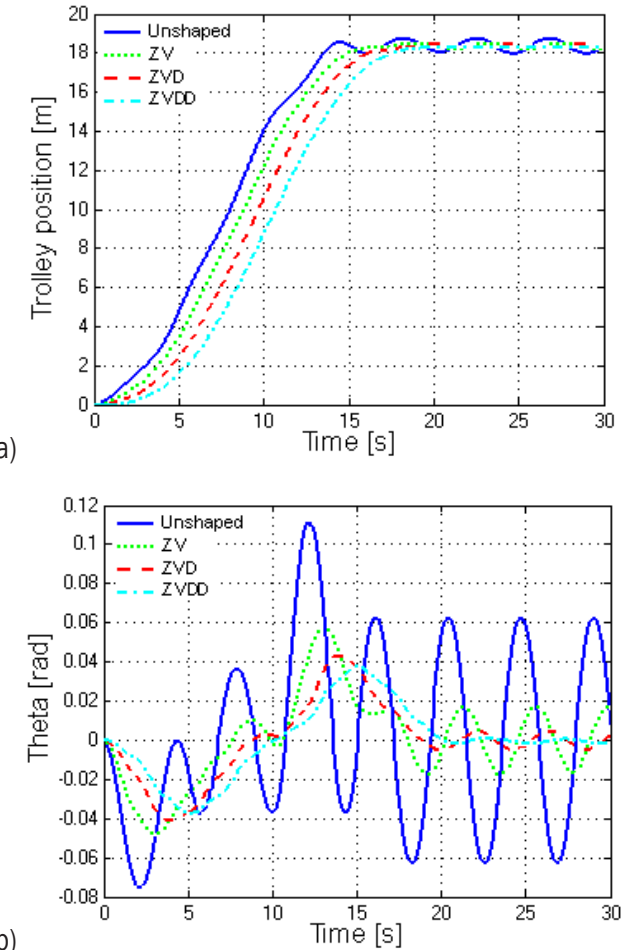

Fig. 9. System response with ZV, ZVD and ZVDD Shaper on changing the length of the hoisting rope and the mass of the payload by 5\%; a) Trolley position and b) load angle with ZV, ZVD and ZVDD Shaper 
The ZVDD shaping method, when compared to the $\mathrm{ZV}$ and the ZVD shaping method, gives the best performance, since oscillations in the steady-state are almost fully cancelled (angular deviation is only $0.001 \mathrm{rad}$ ). The settling time is now $18.5 \mathrm{~s}$. This is 0.75 $\mathrm{s}$ higher than when using the ZVD shaping method.

The length of the hoisting rope and the mass of the payload is always known a-priori on ship-toshore cranes, since the length of the hoisting rope is measured by digital encoders, while the mass of the payload is measured by precise weighting cells. The accuracy of the measurements is $5 \%$. Therefore, it is important to evaluate the robustness of anti-sway systems on smaller variations of parameters. The length of the hoisting rope and the payload mass has been increased by $5 \%$ while keeping the same parameters for ZV, ZVD and ZVDD shapers.

Trolley positions and angles are shown in Figure 9. The angular deviation, when using ZVD shaping method, does not change, while it slightly increases for ZVDD shaping method to $0.0012 \mathrm{rad}\left(0.068^{\circ}\right)$. We could see that both methods are robust to small variations of the length of the hoisting rope and payload mass.

The simulation results show that $\mathrm{ZV}, \mathrm{ZVD}$ and ZVDD shapers are very efficient in reducing oscillations in the system. The operator (on average) stabilises the load within 15 seconds after reaching the final position (separately at ship and at truck location). The ZVD shaping method needs $2.75 \mathrm{~s}$ for stabilisation, while ZVDD method requires 3.5 s. Taking into account that productivity of Panamax ship-to-shore cranes in the Port of Koper is about 19 containers per hour per crane, it can be calculated that the productivity could rise by about 2 containers per hour per crane.

\section{CONCLUSION}

Three open-loop anti-sway systems have been introduced. The systems could be used on Panamax ship-to-shore cranes in the Port of Koper to reduce load oscillations during trans-shipment. The efficiency of the systems was tested on linearized dynamic mathematical model with parameters taken from technical specification of Panamax ship-to-shore crane in Port of Koper. The simulation results show that all three methods reduce load oscillations significantly, but only the ZVD and the ZVDD methods completely satisfy the given requirements. The ZVDD method in comparison to the ZVD method reduces oscillations more efficiently, but results in a slightly slower response. Since ZVD realisation is simpler and faster, it is our preference for anti-sway systems on Panamax ship-to-shore cranes in Port of Koper.

The mathematical model used in the simulation was undamped with a fixed length of the hoisting rope. In our future work, the efficiency of all three anti-sway systems will be tested on a mathematical model with changing hoisting rope length and different damping factors.

\section{REFERENCES}

[1] Omar, H.-M. (2003). Control of Gantry and Tower Cranes. Dissertation for the degree of Doctor of Philosophy in Engineering Mechanics, Blacksburg.

[2] Singer, N.-C. (1989). Residual Vibration Reduction in Computer Controlled Machines. Technical Report 1030, MIT Artificial Intelligence Laboratory, Massachusetts.

[3] Bolz, R.-E., Tuve, G.-L. (1973). CRC Handbook of Tables for Applied Engineering Science. CRC Press, Boca Raton.

[4] Gieck, K. (1983). Engineering Formulas. McGrawHill, New York.

[5] Singer, N.-C., Seering, W.-P. (1990). Preshaping Command Inputs to Reduce System Vibration. Journal of Dynamic Systems, Measurement, and Control, vol. 112, p. 76-82, DOI:10.1115/1.2894142.

[6] Singhose, W., Singer, N., Seering, W. (1995). Comparison of Command Shaping Methods for Reducing Residual Vibration. Proceeding of the 1995 European Control Conference, vol. 2, p. 1126-1131.

[7] Huh, C., Hong, K. (2002) Input shaping control of container crane systems: limiting the transient sway angle. 15th Triennial World Congress, Barcelona.

[8] Dieulot, J. Y., Thimoumi, I., Colas, F., Bearee, R. (2006). Numerical Aspect and Performances of Trajectory Planning Methods of Flexible Axes. Internal Journal of Computers, Communications \& Control, vol. 1 , no. 4 , p. $35-44$.

[9] Pao, Y., Singhose, W. (1995). On the Equivalence of Minimum Time Input Shaping with Traditional Time-Optimal Control. Proceedings of the $4^{\text {th }}$ IEEE Conference on Control Applications, p. 1120-1125. 